\title{
Luminosity monitor using the coherent beam-beam interaction
}

\author{
D. Sagan \\ Laboratory of Nuclear Studies, Cornell University, Ithaca, New York 14853
}

(Received 25 April 2001; published 14 December 2001)

\begin{abstract}
At the Cornell Electron/Positron Storage Ring, a fast luminosity monitor has been implemented. The monitor works by measuring the amplitude of oscillation of selected bunches while the corresponding opposing bunches are shaken. Signal-to-noise ratios of better than 100:1 have been obtained with 1 sec averaging times. A software normalization program ameliorates the unwanted dependence of the signal upon certain vertical lattice parameters such as the vertical tune.
\end{abstract}

PACS numbers: 29.20.Dh, 29.27.Fh, 29.40.Gx

\section{INTRODUCTION}

In a colliding beam storage ring it is essential to be able to monitor the luminosity so as to be able to adjust machine elements (magnets, separators, etc.) to maximize the luminosity. Two methods that are used at the Cornell Electron/Positron Storage Ring (CESR) involve measuring the vertical $\sigma-\pi$ tune split [1] and counting bhabhas [2] using the CLEO detector [3]. The problem with the former method is that the $\pi$ mode is not always cleanly visible on a spectrum analyzer. On the other hand, the latter method is slow since the counting rates are low - the characteristic time scale for a measurement being a minute.

With these problems in mind an alternative method has been developed that uses the coherent beam-beam interaction (BBI) [4]. The monitor is shown schematically in Fig. 1. Selected bunches of one beam are shaken vertically at a fixed frequency. These "shaker bunches" interact with the corresponding opposing bunches of the opposite beam (the "detected bunches") at the interaction point (IP). The oscillations of the detected bunches are monitored, and the amplitude of oscillation of the detected bunches is a mea-

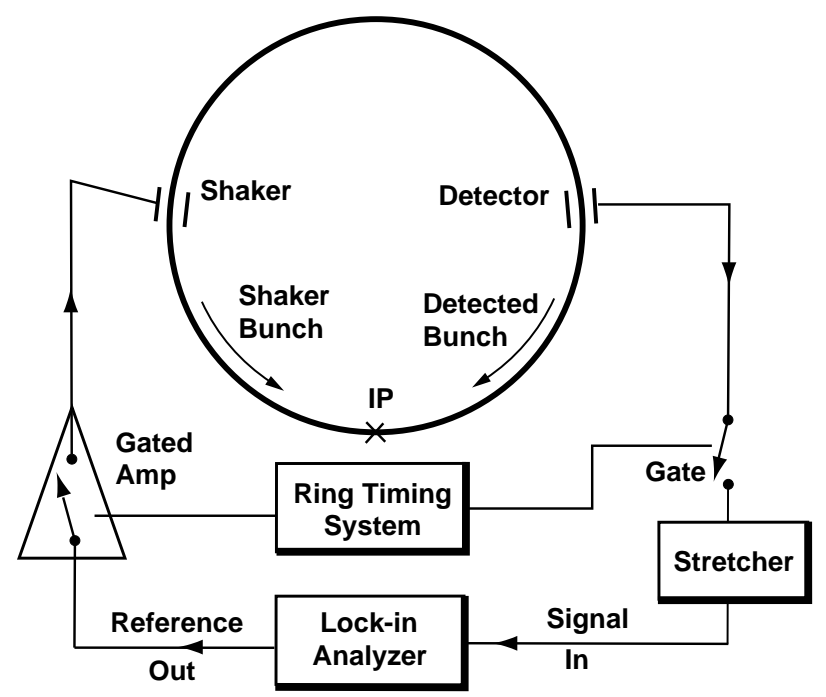

FIG. 1. Schematic diagram of the beam-beam interaction (BBI) luminosity monitor configuration. sure of the luminosity. This beam-beam interaction luminosity monitor has proved to have several advantages: The hardware requirements are minimal and the response is fast with the characteristic time scale for a measurement being about a second. An added benefit is that, with multiple bunches in each beam, it is possible to individually monitor the luminosity of any given pair of colliding bunches, making the study of bunch-to-bunch variations possible.

\section{THEORY}

\section{A. From shaker to detector}

The configuration of the BBI luminosity monitor is shown schematically in Fig. 1. The sinusoidal reference signal at frequency $f_{s}$ from a lock-in amplifier is used to vertically shake a given shaker bunch (or bunches) of one beam with some kick amplitude $K_{s}$. The kicks translate into oscillations at the IP of amplitude $A_{s 0}(\mathrm{IP})$ given by

$$
A_{s 0}(\mathrm{IP})=K_{s} \sqrt{\beta_{y}(\mathrm{sh}) \beta_{y}(\mathrm{IP})} F_{\mathrm{sh}},
$$

where $\beta_{y}$ is the vertical beta function and $F_{\mathrm{sh}} \equiv$ $F\left(\phi_{y}(\mathrm{sh}), Q_{y}, Q_{s}\right)$ is the "transfer function" from the shaker to the IP. As shown in the Appendix, $F_{\text {sh }}$ is dependent upon the phase advance from the shaker to the IP $\phi_{y}(\mathrm{sh})$, the vertical tune $Q_{y}$, and the shaking tune $Q_{s} \equiv$ $f_{s} / f_{\text {rev }}$, where $f_{\text {rev }}$ is the revolution frequency. A necessary condition for the shaking not to affect the luminosity is

$$
A_{s 0}(\mathrm{IP}) \ll \sigma_{y},
$$

where $\sigma_{y}$ is the vertical beam size at the IP. (For compactness, all beam sizes refer to the IP unless explicitly shown otherwise.)

At the IP, the oscillations of the shaker bunch shake the corresponding detected bunch via the beam-beam interaction. The amplitude of the kick given the detected bunch, $K_{d}$, is

$$
K_{d}=A_{s 0}(\mathrm{IP})\left\langle\frac{d y^{\prime}}{d y}\right\rangle_{(x, y)},
$$

where $y^{\prime}$ is the kick given a particle of the detected bunch and $\langle\ldots\rangle$ denotes an average over all the particles with 
the bunch centers displaced with respect to each other by $(x, y)$. The kick from the shaker given to the shaker bunch makes the shaker bunch execute rigid oscillations. As discussed below, the effect of the detected bunch back upon the shaker bunch is negligible compared to the effect of the shaker itself. Thus, the shaker bunch can be treated as rigid and the average kick to the detected bunch can be obtained by a simple integration over the detected bunch. Assuming a Gaussian shape, and taking the shaker and detected bunch sizes to be equal, the average kick is the same as the kick to a single particle with the bunch size scaled by a factor of $\sqrt{2}$ [5]

$$
\left\langle\frac{d y^{\prime}}{d y}\right\rangle_{(x, y)}=\frac{d}{d y} y^{\prime}\left(x, y ; \sqrt{2} \sigma_{x}, \sqrt{2} \sigma_{y}\right) .
$$

With head-on collisions $\left\langle d y^{\prime} / d y\right\rangle$ reaches its maximum value given by

$$
\left\langle\frac{d y^{\prime}}{d y}\right\rangle_{(0,0)}=\frac{2 \pi \xi_{y}}{\beta_{y}(\mathrm{IP})}
$$

where $\xi_{y}$ is the beam-beam tune shift parameter.

From the kick $K_{d}$ that the detected bunch gets, the amplitude of oscillation $A_{d}($ det $)$ of the detected bunch at the beam position monitor (BPM) detector is

$$
A_{d}(\mathrm{det})=K_{d} \sqrt{\beta_{y}(\mathrm{IP}) \beta_{y}(\mathrm{det})} F_{\mathrm{det}},
$$

where $F_{\mathrm{det}} \equiv F\left(\phi_{y}(\mathrm{det}), Q_{y}, Q_{s}\right)$ is the transfer function from the IP to the detector and is dependent upon the $Q_{y}$, $Q_{s}$ and the phase advance between the IP and the detector $\phi_{y}(\mathrm{det})$. Combining Eqs. (1), (3), and (6) gives

$$
A_{d}(\mathrm{det})=K_{s} \beta_{y}(\mathrm{IP})\left\langle\frac{d y^{\prime}}{d y}\right\rangle \sqrt{\beta_{y}(\mathrm{sh}) \beta_{y}(\mathrm{det})} F_{\mathrm{sh}} F_{\text {det }} .
$$

Using Eq. (5) in (7) we have for head-on collisions

$$
A_{d}(\text { det })=2 \pi K_{s} \xi_{y} \sqrt{\beta_{y}(\mathrm{sh}) \beta_{y}(\mathrm{det})} F_{\mathrm{sh}} F_{\text {det }} .
$$

Equations (7) and (8) are not quite correct since the effect of the detected bunch back upon the shaker bunch has been neglected. Including this effect, the amplitude of the shaker bunch at the IP is

$$
A_{s}(\mathrm{IP})=A_{s 0}(\mathrm{IP})+\left\langle\frac{d y^{\prime}}{d y}\right\rangle \beta_{y}(\mathrm{IP}) F_{\mathrm{IP}} A_{d}(\mathrm{IP}),
$$

where $A_{s 0}(\mathrm{IP})$ is given by Eq. (1), and $F_{\mathrm{IP}} \equiv F\left(0, Q_{y}, Q_{s}\right)$ is the transfer function from the IP back to the IP. The amplitude of motion of the detected bunch at the IP, $A_{d}(\mathrm{IP})$, can be written in terms of the amplitude of the shaker bunch using the analog of Eq. (3),

$$
A_{d}(\mathrm{IP})=\left\langle\frac{d y^{\prime}}{d y}\right\rangle \beta_{y}(\mathrm{IP}) F_{\mathrm{IP}} A_{s}(\mathrm{IP}) \text {. }
$$

Combining Eqs. (9) and (10) then gives

$$
A_{s}(\mathrm{IP})=\frac{A_{s 0}(\mathrm{IP})}{1-\left[\left\langle\frac{d y^{\prime}}{d y}\right\rangle \beta_{y}(\mathrm{IP}) F_{\mathrm{IP}}\right]^{2}} .
$$

The denominator on the right-hand side of Eq. (11) gives the correction due to the effect of the detected bunch back upon the shaker bunch. Using Eq. (5), the maximum correction due to this effect is $\left(2 \pi \xi_{y} F_{\mathrm{IP}}\right)^{2}$. Since $\xi_{y}$ is limited to about 0.05 for existing machines, and since away from a resonance $F$ is of order unity (cf. the Appendix), the effect is of order $10 \%$ and so can be neglected in a first approximation. The exceptional case where the shaking is near resonance will be ignored since, as explained below, this is an undesirable operating condition.

With multiple bunches in each beam, the oscillations of one bunch may be transmitted to another bunch via the long-range BBI at a parasitic crossing point. The analysis of this is similar to the above except here the long-range $\xi_{y}$ appropriate for the particular parasitic crossing point must be used [cf. Eq. (8)]. Since the long-range $\xi_{y}$ are typically an order of magnitude smaller than $\xi_{y}$ at the IP, this effect can also be ignored to first order.

\section{B. Signal processing}

The signal from the detected bunch is rectified, stretched, and held until the signal from the next detected bunch is received. The stretched signal is monitored by the lock-in amplifier which acts as a precision filter. In order to prevent unwanted interference, the shaker is gated so as to only kick the appropriate shaker bunches. Additionally, the signal from the BPM is gated before the stretcher to exclude any direct signal from the shaker bunches.

For the luminosity monitor to be useful, the signal-tonoise ratio must be reasonably large. To analyze this, the model circuit for the detector system shown in Fig. 2 is used. The bipolar signal from a button electrode is split between a load resistor $R_{L}$ (used for circuit protection and to prevent multiple reflections) and a coaxial cable. Only the signal from the correct bunch is allowed to pass through the gate. The signal is rectified by a diode, and the rectified signal is used to charge a capacitor. The voltage on the capacitor is captured by the unity gain sample-and-hold buffer. The signal is then sent via a filter to the lock-in

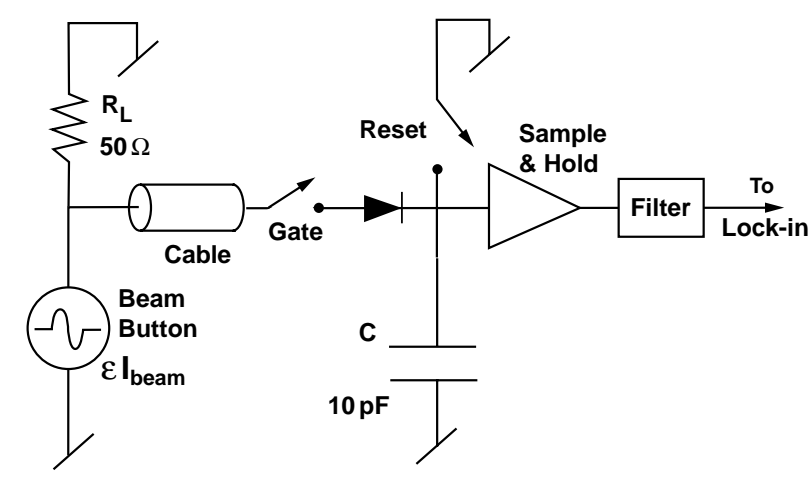

FIG. 2. Model circuit for the electronics between the beam button pickups and the lock-in amplifier detector. There are two such circuits in use. One for the top and the other for the bottom button electrodes. 
amplifier. The filter is used so as to not overload the lock-in front end. Just before the signal from the next sampled bunch reaches the capacitor, the capacitor is discharged by the reset switch and the process repeats itself. In order to double the signal, two identical circuits are used: one using the top and the other using the bottom button electrodes of the BPM. Both signals are fed into the lock-in amplifier which takes the difference between the two.

The signal from the beam is a bipolar pulse. As long as the bunch length $\sigma_{z}$ is less than the diameter of the button electrode, the positive going part of the signal can be approximated by

$$
I_{\mathrm{sig}} \approx \epsilon_{\mathrm{BPM}} I_{b},
$$

where $I_{b}$ is the beam current and $\epsilon_{\mathrm{BPM}}$ is a geometric factor dependent upon the chamber and monitor electrode geometry and the position of the beam. Assuming that half the signal is absorbed by the load resistor and the other half charges the capacitor, the voltage $V_{c}$ on the capacitor is

$$
V_{c}=\frac{\epsilon_{\mathrm{BPM}} N_{b} e}{2 C},
$$

where $N_{b}$ is the number of particles in a bunch, and $C$ is the capacitance. At the lock-in amplifier the rms voltage signal $V_{\text {sig }}$ due to vertical oscillations in the beam is then

$$
V_{\mathrm{sig}}=\frac{N_{b} e}{C} \frac{\partial \epsilon_{\mathrm{BPM}}}{\partial y} \frac{A_{d}(\mathrm{det})}{\sqrt{2}},
$$

where a factor of $\sqrt{2}$ comes from converting peak amplitude to rms and another factor of 2 comes from using the difference signal between the top and bottom electrodes.

The noise voltage $V_{n}$ is calculated by first considering the rms noise voltage $V_{n}$ (cap) at the capacitor due to the load resistor [6],

$$
V_{n}^{2}(\operatorname{cap})=\frac{k_{B} T}{C}\left(1-e^{-2 t_{c} / \tau}\right),
$$

where $k_{B}$ is Boltzmann's constant, $T$ is the temperature of the resistor, $\tau=R C$ is the charging time constant, and $t_{c}$ is the charging time of the capacitor (the time the diode in Fig. 2 is conducting). At the detector the duration of the signal is of order $c / 2 \sigma_{z}$. With any dispersion in the cable connecting the BPM to the diode the signal is stretched giving a larger $t_{c}$. It is assumed here (consistent with CESR monitor parameters) that $t_{c} \gg \tau$ so that from Eq. (15) $V_{n}$ (cap) is given by

$$
V_{n}(\mathrm{cap})=\sqrt{\frac{k_{B} T}{C}} .
$$

It is assumed that the sample-and-hold and filter do not introduce any appreciable noise so that Eq. (16) gives the noise at the input to the lock-in amplifier as well. At the output of the lock-in amplifier the noise is reduced since the lock-in averages the signal over some time period. The effective number of samples $N_{s}$ within the averaging period is related to the lock-in equivalent noise bandwidth $f_{n}$ by

$$
N_{s} \simeq \frac{1}{f_{n} t_{b b}}
$$

where $t_{b b}$ is the time between sampled bunches. Since the lock-in amplifier samples $N_{s}$ times, the noise on the output from the lock-in will be reduced by $\sqrt{N_{s}}$. From Eq. (16) the lock-in output signal will thus have an rms noise of

$$
V_{n} \simeq \sqrt{\frac{2 k_{B} T f_{n} t_{b b}}{C}},
$$

where the extra factor of $\sqrt{2}$ comes from using the difference signal between top and bottom electrodes. $f_{n}$ can be related to the settling time of the lock-in amplifier. For example, for a simple RC filter, as used in the CESR monitor lock-in, $f_{n}$ may be approximated by

$$
f_{n} \simeq \frac{1}{t_{1}}
$$

where $t_{1}$ is the time for the response to settle to within $1 \%$ of its final value after a step change in signal.

\section{Luminosity versus monitor signal}

The BBI luminosity monitor is useful for maximizing the luminosity by maintaining head-on collisions. This is illustrated in Fig. 3 which shows $\left\langle y^{\prime}\right\rangle,\left\langle d y^{\prime} / d y\right\rangle$ (which, with the lattice functions fixed, is proportional to the monitor signal) and $\mathcal{L}$ as a function of $y / \sigma_{y}$ with $x=0$. The kick $y^{\prime}$ was calculated using the standard Bassetti and Erskine complex error function formula [1] with, typical for CESR, a value of $\sigma_{y} / \sigma_{x}=0.01 . \mathcal{L}$ was calculated from the standard overlap formula

$$
\mathcal{L}=\mathcal{L}_{0} e^{-y^{2} / 4 \sigma_{y}^{2}} .
$$

Both $\mathcal{L}$ and $\left\langle d y^{\prime} / d y\right\rangle$ are maximal for $y=0$ and, surprisingly enough, for offsets of less than $2 \sigma_{y}$ the luminosity and $\left\langle d y^{\prime} / d y\right\rangle$ are simply proportional to one another. Thus,

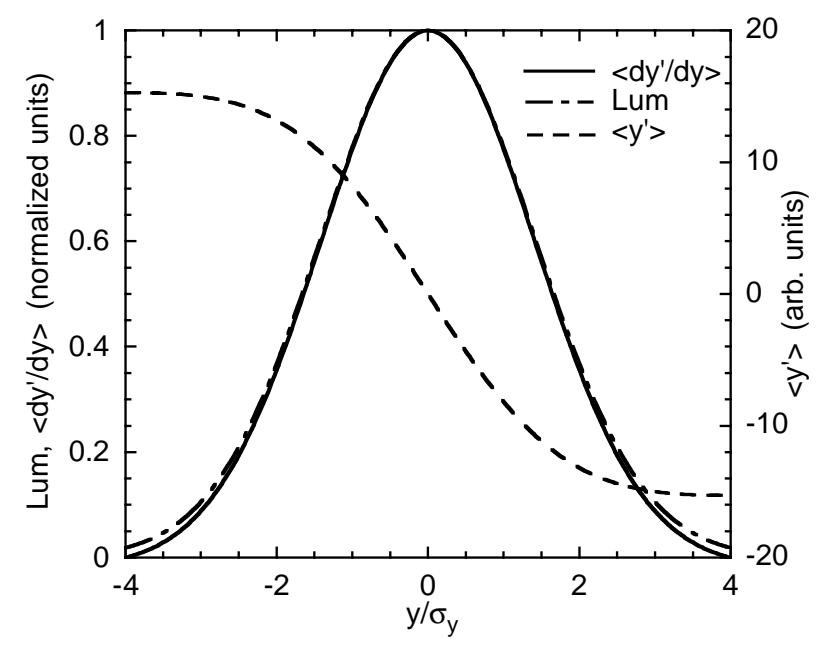

FIG. 3. $\mathcal{L},\left\langle d y^{\prime} / d y\right\rangle$, and $\left\langle y^{\prime}\right\rangle$ as a function of $y / \sigma_{y}$ for $\sigma_{y} / \sigma_{x}=0.01$ and $x=0 . \mathcal{L}$ and $\left\langle d y^{\prime} / d y\right\rangle$ have been scaled to be 1 at $y=0$. 
with respect to variations of $y$, the monitor signal is a direct measure of the luminosity.

Besides using the BBI luminosity monitor to center the beams, the monitor can be used to maximize the luminosity by minimizing the horizontal/vertical beam coupling and hence minimizing the vertical beam size. This is true since, from Eq. (7), the luminosity signal is proportional to $\xi_{y}$, and $\xi_{y}$ is related to the beam sizes through the standard formula

$$
\xi_{y}=\frac{N_{b} \beta_{y}(\mathrm{IP}) r_{e}}{2 \pi \gamma \sigma_{y}\left(\sigma_{x}+\sigma_{y}\right)},
$$

with $\gamma$ being the standard relativistic factor, and $r_{e}$ the classical electron radius. $\xi_{y}$ can also be related to the luminosity by

$$
\mathcal{L}=\frac{\gamma I \xi_{y}}{2 e r_{e} \beta_{y}(\mathrm{IP})}(1+r),
$$

where $e$ is the electron charge, $I$ is the beam current, and $r \equiv \sigma_{y} / \sigma_{x}$. As long as $\beta_{y}$ is held constant, the monitor signal will track the luminosity with variations in beam size.

\section{Normalization program}

The BBI luminosity monitor signal has the same problem as one has in using the $\sigma-\pi$ tune split to monitor the luminosity. That is, at constant luminosity, the monitor signal is dependent upon the vertical tune, the vertical phase advance between the IP, shaker, and detector, as well as the vertical betas at these points. An example of this is shown in Fig. 4. Figure 4 shows the monitor signal $S$ [proportional to $A_{d}(\mathrm{det})$ ], as calculated from Eq. (7), as a function of vertical tune while holding the luminosity and the horizontal tune constant. Parameters used in the computation are typical of CESR high energy physics (HEP) conditions and are given in Table I. The change in $Q_{y}$ is simulated by varying the strengths of the quadrupoles in the arcs to give

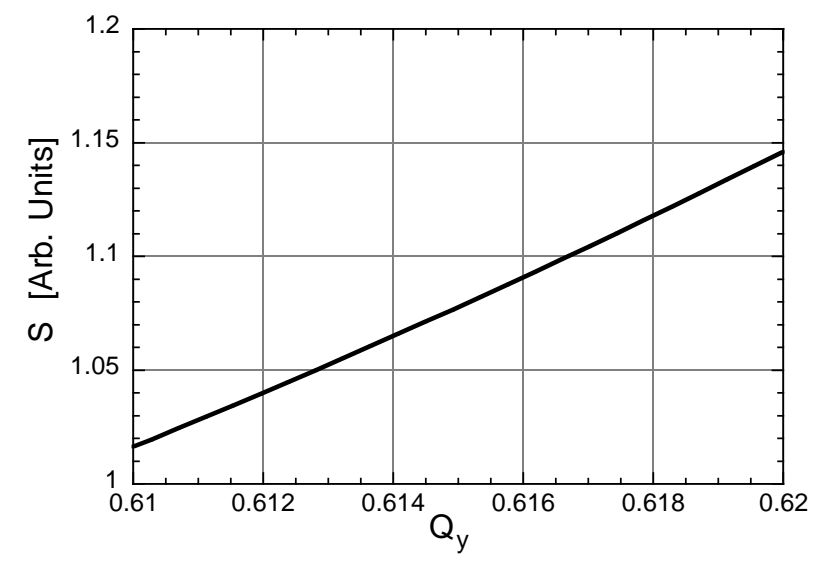

FIG. 4. Variation of $S$ as a function of vertical tune with the luminosity and horizontal tune held constant.
TABLE I. CESR BBI luminosity monitor parameters.

\begin{tabular}{cc}
\hline \hline Parameter & Value \\
\hline$I$ & $6 \mathrm{~mA}$ \\
$\delta$ & $10^{-4}$ \\
$Q_{y}$ & 9.596 \\
$Q_{s}$ & $0.26(100 \mathrm{kHz})$ \\
$f_{\text {rev }}$ & $390 \mathrm{kHz}$ \\
$K_{s}$ & $0.3 \mu \mathrm{rad}$ \\
$\xi_{y}$ & 0.03 \\
$r$ & 0.01 \\
$N_{b}$ & $10^{11}$ \\
$C$ & $10 \mathrm{pF}$ \\
$t_{1}$ & $1 \mathrm{sec}$ \\
$\partial \epsilon_{\text {BPM }} / \partial y$ & $0.64 / \mathrm{m}$ \\
$\sigma_{y}(\mathrm{det})$ & $300 \mu \mathrm{m}$ \\
$\sigma_{y}(\mathrm{IP})$ & $7 \mu \mathrm{m}$ \\
$\sigma_{z}$ & $1.8 \mathrm{~cm}$ \\
$N_{\text {bunnh }}$ & 9 \\
$\beta_{y}(\mathrm{IP})$ & $0.019 \mathrm{~m}$ \\
$\beta_{y}(\mathrm{sh})$ & $21.5 \mathrm{~m}$ \\
$\beta_{y}(\mathrm{det})$ & $32.3 \mathrm{~m}$ \\
$\phi_{y}(\mathrm{sh})$ & $2 \pi \times 0.86$ \\
$\phi_{y}(\mathrm{det})$ & $2 \pi \times 0.27$ \\
$\left|F_{\text {sh }}\right|$ & 0.79 \\
$\left|F_{\text {det }}\right|$ & 0.82 \\
$\left|F_{\text {IP }}\right|$ & 0.44 \\
\hline \hline
\end{tabular}

minimal change in the beta functions. Over the tune range of the graph, which is typical of the range over which the tune can be varied during a HEP run, $S$ varies by of order $10 \%$. This variation can be larger than the actual variation of the luminosity. The trend of $S$ in the figure can be simply understood as a resonance phenomena by noting that, as the tune is increased, the aliased tune, $1-Q_{y}$, is moving nearer the shaking frequency at $Q_{s}=0.26$.

In order to overcome the dependency of the monitor signal on the vertical lattice functions, a software program has been developed that normalizes the signal to give a quantity that, at least in theory, is directly a measure of the specific luminosity. The normalization program works by using Eq. (7) to compute the "predicted" signal $S_{p}$ assuming constant $\mathcal{L} / I$. With this, the normalized signal $S_{n}$ is given by

$$
S_{n} \equiv \frac{S}{S_{p} I} .
$$

At the start of a HEP run, the values for the quadrupole strengths, quadrupole rotation angles, and sextupole strengths [7] are read in from the CESR database. It is assumed that these initial settings correspond to the settings for the theoretical design lattice. That is, the initial Twiss parameters are assumed equal to the Twiss parameters of the design lattice. $S_{p}$ is computed assuming a constant value for $\mathcal{L} / I$ with head-on collisions.

The program recomputes $S_{n}$ approximately 8 times a second and puts this number in the CESR database for 


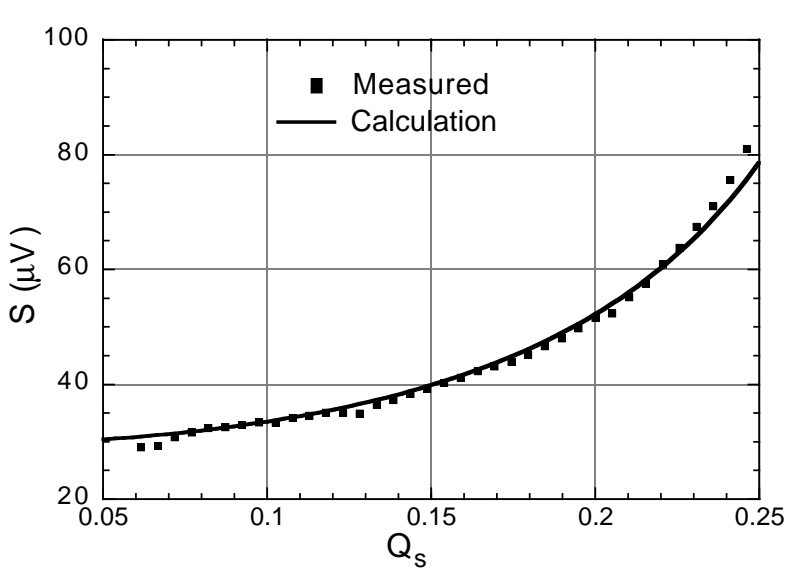

FIG. 5. Calculated and measured monitor signals as a function of shaker frequency. With the calculated $S$ there is one adjustable constant that gives the overall gain of the system. This constant was chosen to best match the measured results.

general use. To save on computation time, the database is monitored, and only when a quadrupole or sextupole setting is changed are the Twiss parameters and $S_{p}$ recomputed. This recomputation is based upon the change in the settings and the known calibration constants.

Figure 5 shows a test of the normalization program showing the measured and calculated monitor signals as a function of vertical tune. There is good agreement between the two. The greatest weakness in the above procedure is the assumption that the initial conditions correspond to the design lattice. This assumption is necessary since hysteresis in the magnets precludes an accurate enough absolute calibration. In practice, the assumption is generally close enough so that the normalized signal generally follows the luminosity and thus the program is a significant help in tuning the CESR ring [8]. One way to desensitize the signal dependence on tune would be to lower $Q_{s}$, as shown in Fig. 6. Figure 6 shows, as a function of $Q_{s}$, the quantity $d S / S$ - the change in $S$, for a fixed change in $Q_{y}$, nor-

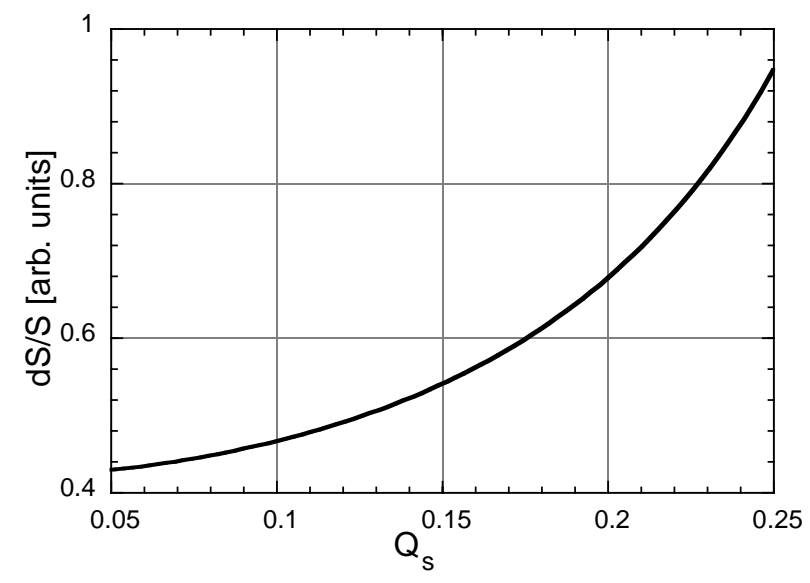

FIG. 6. Relative sensitivity of the monitor signal to changes in vertical tune as a function of shaker frequency. $d S$ is the change in $S$, for a fixed change in $Q_{y}$, as computed from Eq. (7). malized by $S$. Lowering $Q_{s}$, however, has the drawback of lowering $S$ (cf. Fig. 5) since, currently at CESR, the shaker driver amplifier is near maximum output and so $K_{s}$ cannot be increased. Moreover, lowering $Q_{s}$ does nothing to lessen the dependence on the betas.

\section{THE CESR BBI LUMINOSITY MONITOR}

\section{A. Signal and noise}

Typical values for the parameters of the CESR BBI luminosity monitor are given in Table I [9]. For CESR, the shaker kick $K_{s}$ is limited to the value given in Table I by the shaker power amplifier. This being the case, the shaking frequency $Q_{s}$ is chosen as a compromise between being far enough from a betatron resonance so that the monitor signal is not too sensitive to changes in the vertical tune while keeping close enough to a resonance to have a reasonable signal amplitude. With the numbers given in Table I, and Eq. (1), the shaker bunch has an oscillation amplitude at the IP of

$$
\left|A_{s 0}(\mathrm{IP})\right|=0.15 \mu \mathrm{m} .
$$

This is 3\% of $\sigma_{y}$ so Eq. (2) is satisfied. From Eqs. (3) and (5) this translates, when the bunches collide head-on, to a kick of the detected bunch of

$$
\left|K_{d}\right|=1.8 \mu \mathrm{rad} .
$$

The amplitude of the detected-bunch oscillations at the IP is then

$$
\left|A_{d}(\mathrm{IP})\right|=\left|K_{d} \beta_{y}(\mathrm{IP}) F_{\mathrm{IP}}\right|=15 \mathrm{~nm} .
$$

That $A_{d}(\mathrm{IP}) \ll A_{s}(\mathrm{IP})$ shows that the effect of the detected bunch back on the shaker bunch can be ignored. Using Eq. (6) with the value for $K_{d}$ gives the amplitude of the detected bunch at the detector

$$
\left|A_{d}(\mathrm{det})\right|=0.9 \mu \mathrm{m} .
$$

Using this in Eq. (14) gives the lock-in amplifier voltage signal

$$
V_{\text {sig }}=630 \mu \mathrm{V} \text {. }
$$

Measurements of the lock-in voltage give the result

$$
V_{\text {sig }}=170 \mu \mathrm{V} \text {. }
$$

Considering the crudeness of the model of the detector electronics, the factor of 4 difference between the calculation and measurement is not unreasonable. A contributing factor to the difference is the attenuation in the cable which was not taken into account. Another problem is that the capacitance shown in Fig. 2 is due to the parasitic front end capacitance of the buffer and therefore the value listed in Table I is only an estimate.

Using Eq. (18) with the values listed in Table I gives the noise voltage at the output of the lock-in to be 


$$
V_{n}=\frac{0.05 \mu \mathrm{V}}{\sqrt{\mathrm{Hz}}} \sqrt{f_{n}} .
$$

A direct measurement, on the other hand, gives a much larger number,

$$
V_{n} \simeq \frac{0.7 \mu \mathrm{V}}{\sqrt{\mathrm{Hz}}} \sqrt{f_{n}} .
$$

Why the measured noise is much larger than the theoretical noise is not clear. Without a beam to drive the rectifying diode into its conducting region the noise is practically eliminated indicating that the electronics is not a source of the noise. In any case, with the experimentally measured numbers, if one chooses a $t_{1}$ of $1 \mathrm{sec}$, which is equivalent to a bandwidth of $1 \mathrm{~Hz}$ [cf. Eq. (19)] the measured signal-tonoise ratio is greater than 100 to 1 .

\section{B. Experimental results}

To directly compare the monitor signal with the luminosity, the monitor signal and the luminosity, as measured by the CLEO detector, were measured as functions of relative bunch vertical displacement $y$. $y$ was varied using electrostatic separators which differentially steer the opposing bunches. Figure 7 shows the results. A linear fit to the data shows good agreement. Also shown in the figure is the theoretical curve, using the results of Fig. 3, scaled so that the data point of maximum $\mathcal{L}$ is taken to be at $y=0$. There is a discrepancy between the slopes of the data and the theoretical curves. This cannot be explained by a dependence of $\sigma_{y}$ on $y$ since varying $\sigma_{y}$ would give a linear relationship of monitor signal and $\mathcal{L}$ very nearly equal to theory.

In CESR the bunches in a beam are clustered in "trains." The bunches within a train are called "cars." One of the advantages of the luminosity monitor is the ability to measure the luminosity of individual bunches and thus it is possible

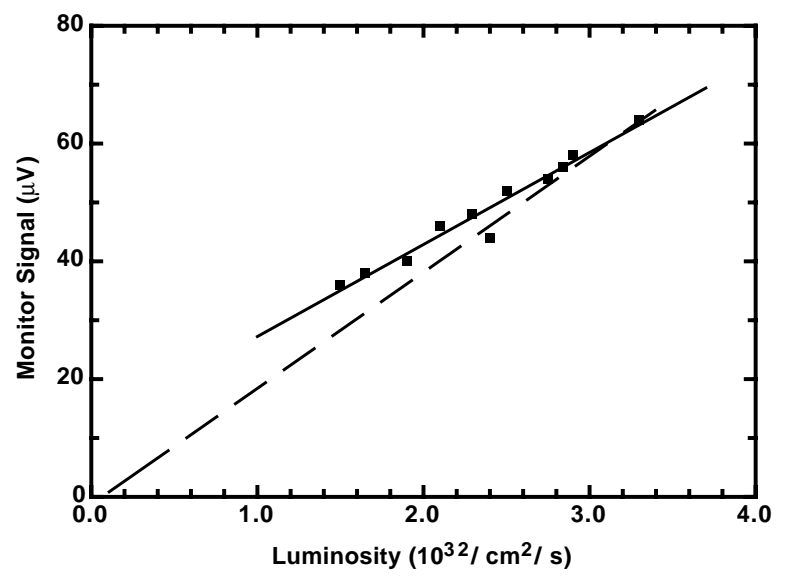

FIG. 7. Car \#2 monitor signal as a function of CLEO luminosity while varying the vertical bunch offset $y$. Squares: data points; solid line: linear fit to the data; dashed line: theory scaled so that the data point with maximum $\mathcal{L}$ is assumed to be at $y=0$.

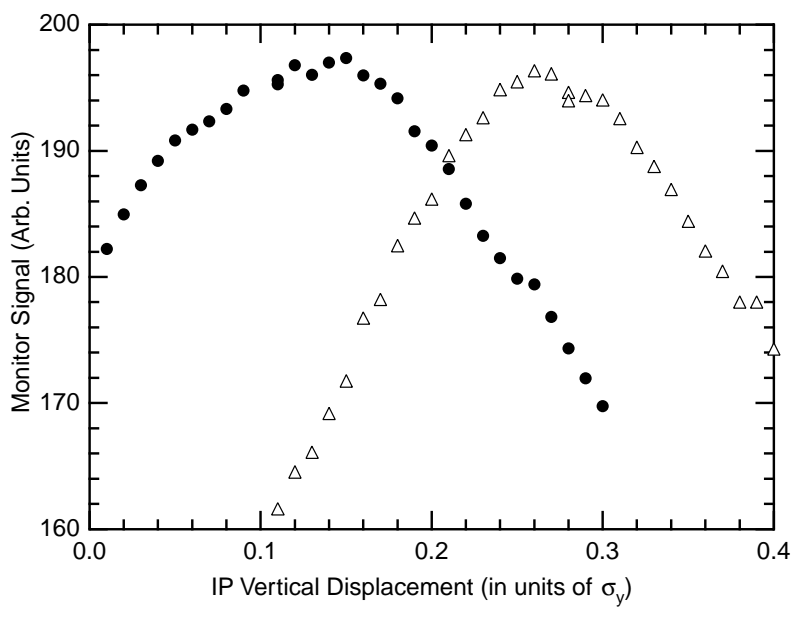

FIG. 8. Monitor signals for cars \#2 and \#5 as a function of vertical displacement of the bunches.

to see bunch-to-bunch effects. This is shown in Fig. 8 which shows the monitor signal for cars \#2 and \#5 of a five car train. In this experiment, the car-to-car spacing within a train was $14 \mathrm{nsec}$. In all there were nine trains which were spaced about 200 nsec apart. Figure 8 shows the monitor signal as a function of $y$ normalized in units of $\sigma_{y}$ appropriate for the observed luminosity. The fact that the peaks of the two signals do not coincide implies that the cars are not following the same vertical trajectory. This phenomena has so far been unexplained, but one possible suspect is the short range wake fields produced by the leading (\#2) car.

\section{CONCLUSION}

Using the coherent beam-beam interaction to monitor the luminosity has several clear advantages: The system has a fast response time so tuning of machine elements can be done efficiently by an operator or a computer program. The system is also easy to construct - the necessary shaker and detector hardware are typical of any storage ring and the external electronics is minimal. Additionally, bunch-to-bunch variations in the luminosity can be monitored. Problems with the monitor signal being dependent upon the vertical lattice functions as well as the luminosity can be ameliorated using a normalization software program.

\section{ACKNOWLEDGMENTS}

A debt of gratitude is owed to John Sikora and Stu Henderson for helping put the luminosity monitor together and to Gerry Codner for help with the electronics. Additionally, thanks must go to Bob Meller, Mike Billing, and Don Hartill for useful discussions. Last but not least, thanks also must be given to Elizabeth Young and Richard Talman whose work on measuring the beam-beam kick was the inspiration that started the whole process. 


\section{APPENDIX: RESPONSE TO A SHAKER}

The object is to calculate the beam oscillations induced by a shaker. The coordinates $\left(y, y^{\prime}\right)$ of the beam at some longitudinal position can be described using a complex number $z$,

$$
z \equiv y-i y^{\prime} \beta_{y}+i \alpha_{y} y
$$

where $\beta_{y}$ and $\alpha_{y}$ are Twiss parameters. Let $z(s, n)$ denote the coordinates on turn $n$ and at longitudinal position $s$. Without shaking, the free oscillations of the beam can be described by

$$
z(s, n)=z\left(s_{\mathrm{sh}}, m\right) \sqrt{\frac{\beta_{y}(s)}{\beta_{y}(\mathrm{sh})}} e^{2 \pi i Q_{y}(n-m)} e^{-\delta(n-m)} e^{i \phi_{y}(s)},
$$

where $\delta$ is the damping decrement per turn (which is assumed to be small compared to unity), $\phi_{y}(s)$ is the phase advance from the shaker at position $s_{\text {sh }}$ to position $s$, and $Q_{y}$ is the vertical tune. The kick given by the shaker on turn $m$ is

$$
\delta z\left(s_{\mathrm{sh}}, m\right)=-i \beta_{y}(\mathrm{sh}) \operatorname{Re}\left[K_{s} e^{2 \pi i Q_{s} m}\right],
$$

where Re denotes the real part and $Q_{s}$ is the frequency of the shaker. In general, the shaking amplitude $K_{s}$ may be complex. To simplify the derivation it is here, without loss of generality, taken to be real so that

$$
\delta z\left(s_{\mathrm{sh}}, m\right)=-i K_{s} \beta_{y}(\mathrm{sh}) \cos \left(2 \pi Q_{s} m\right) .
$$

With the shaker turned on, $z(s, n)$ can be written as the sum of the displacements due to the kicks on all the previous turns. Using Eqs. (A2) and (A4) thus gives

$$
\begin{aligned}
z(s, n) & =-i K_{s} \sqrt{\beta_{y}(s) B_{y}(\mathrm{sh})} \sum_{m=-\infty}^{n} \cos \left(2 \pi Q_{s} m\right) e^{2 \pi i Q_{y}(n-m)} e^{-\delta(n-m)} e^{i \phi_{y}(s)} \\
& =\frac{-i K_{s} \sqrt{\beta_{y}(s) \beta_{y}(\mathrm{sh})}}{2}\left[\frac{e^{i\left[\phi_{y}(s)+2 \pi Q_{s} n\right]}}{1-e^{2 \pi i\left(Q_{y}-Q_{s}\right)} e^{-\delta}}+\frac{e^{i\left[\phi_{y}(s)-2 \pi Q_{s} n\right]}}{1-e^{2 \pi i\left(Q_{y}+Q_{s}\right)} e^{-\delta}}\right]
\end{aligned}
$$

The position $y$ is given by the real part of $z(s, n)$, and from Eq. (A5) $y$ can be written in the form

$$
y=\operatorname{Re}\left[A_{y} e^{2 \pi i Q_{s} n}\right] .
$$

The amplitude of the beam $A_{y}$ can be related to $K_{s}$ via

$$
A_{y} \equiv K_{s} \sqrt{\beta_{y}(\mathrm{sh}) \beta_{y}(s)} F .
$$

This defines $F$. Using Eq. (A5) with Eq. (A7) gives

$$
\begin{aligned}
F=\frac{-i}{2} & {\left[\frac{e^{i \phi_{y}(s)}}{1-e^{2 \pi i\left(Q_{y}-Q_{s}\right)} e^{-\delta}}\right.} \\
& \left.-\frac{e^{-i \phi_{y}(s)}}{1-e^{-2 \pi i\left(Q_{y}+Q_{s}\right)} e^{-\delta}}\right] .
\end{aligned}
$$

$F$ is a complex quantity. The magnitude of $F$ gives the magnitude of the $y$ oscillations normalized to the shaking amplitude. The phase of $F$ gives the phase of the oscillations relative to the shaker. $|F|$ is of order unity except near a resonance where $Q_{s} \approx k \pm Q_{y}$ for some integer $k$. Near a resonance, $F$ has the standard form for a resonant oscillator,

$$
F \simeq \frac{ \pm 1}{2} \frac{e^{ \pm i \phi_{y}(s)}}{2 \pi\left(k \pm Q_{y}-Q_{s}\right)+i \delta} .
$$

In the case where $Q_{s}$ is near an integer, but $Q_{y}$ is not, $F$ has the same form as a static orbit bump,

$$
F \simeq \frac{\cos \left[\phi_{y}(s)-\pi Q_{y}\right]}{2 \sin \pi Q_{y}}
$$

[1] R. Talman, in Physics of Particle Accelerators, edited by M. Month and M. Dienes, AIP Conf. Proc. No. 153 (AIP, New York, 1987), pp. 789-834.

[2] H. J. Bhabha, Proc. R. Soc. London A 154, 195 (1935).

[3] G. Crawford et al., Nucl. Instrum. Methods Phys. Res., Sect. A 345, 345 (1994).

[4] The original article describing the luminosity monitor was D. Sagan, J. Sikora, and S. Henderson, in Proceedings of the 1997 Particle Accelerator Conference, Vancouver, Canada (IEEE, Piscataway, NJ, 1998), p. 1765; a followup article describing the normalization program was D. Sagan, in Proceedings of the 1999 Particle Accelerator Conference, New York (IEEE, Piscataway, NJ, 1999), p. 2969.

[5] K. Hirata, Nucl. Instrum. Methods Phys. Res., Sect. A 269, 7-22 (1988).

[6] F. Reif, Fundamentals of Statistical and Thermal Physics (McGraw-Hill, New York, 1965).

[7] Since a pretzeled orbit is used to separate the beams (which share a common beam pipe), the sextupoles affect the Twiss parameters and thus need to be monitored.

[8] John Hylas (private communication).

[9] The value for $\partial \epsilon_{\mathrm{BPM}} / \partial y$ is obtained from Mike Billing, CESR Internal Note No. 7/4/80, 1980. 